\title{
EDITORIAL
}

\section{FEVER THERAPY IN THE VENEREAL DISEASES}

\author{
1
}

The indication for artificial fever in the treatment of syphilis and gonorrhoea derives from the observation that the temperature of the patient can be raised to and be maintained at the thermal death rate of the Spirochaeta pallida or of the gonococcus without at the same time causing injury to any of the body tissues and without involving serious risk of a fatal issue owing to circulatory failure or heatstroke. Nevertheless clinical experience demonstrates that while there are clear-cut indications for fever therapy, there are also well-defined limitations to its use, and that the treatment of the common venereal diseases is not a field for the indiscriminate application of hyperthermia. Uncomplicated gonorrhoea responds in at least 90 per cent of cases to properly applied chemotherapy with sulphonamides, and in early syphilis the use of the arsphenamines and bismuth holds out an excellent prospect of cure ; neither the early uncomplicated stages of these diseases nor the chemotherapy employed in either respectively, involves any appreciable risk or immediate threat to life. Obviously the same relative absence of immediate danger cannot be claimed for a procedure which raises the patient's 'temperature to levels in the neighbourhood of $106^{\circ} \mathrm{F}$. and maintains it at these levels for many hours. The wisdom of employing a " cure " which may be more dangerous than the disease itself is obviously open to challenge.

Boak and her colleagues estimated the in vitro thermal death time of many different strains of gonococci and determined that, at a temperature of $105 \cdot 8^{\circ} \mathrm{F}$. 99 per cent of the gonococci were killed in from four to five hours ; the remaining 1 per cent showed a varying higher degree of heat resistance. These workers showed that at a temperature of $102 \cdot 2^{\circ} \mathrm{F}$. the growth of the gonococcus was not appreciably affected. It is clear that inoculation methods of inducing fever, such as the commonly used intravenously administered vaccines T.A.B., B. Coli and Dmelcos, or injections of foreign protein such as milk, or chemical agents, are all likely to be attended with limited success. Methods of this kind may help to enhance and mobilize the defence mechanisms by inducing a leucocytosis or by promoting increased antibody formation, but they cannot readily achieve a sufficiently high and sustained pyrexia. These inoculation methods used in conjunction with the sulphonamides may aid the action of the chemotherapy, but such aid is relatively weak when compared with the efficacy of the physical methods of producing fever.

The experiments of Boak and her colleagues placed the thermal death point of cultures of $S$. pallida in vitro at $105.9^{\circ} \mathrm{F}$. when maintained for one hour, but it is difficult to evaluate such findings in respect of $S$. pallida, in view of the uncertainty regarding cultures. Bessemans and others established that rabbit chancres resolve when the temperature of the tissue is maintained at $104.9^{\circ} \mathrm{F}$. for one hour. Nevertheless, the work of Neymann and others on the treatment of human primary and secondary syphilis showed that a temperature of $107 \cdot 6^{\circ} \mathrm{F}$. sustained for several hours or of $105 \cdot 8^{\circ} \mathrm{F}$. sustained for from ten to fifteen hours cannot be relied upon to kill all the $S$. pallida in the lymphatic glands. These last investigations demonstrated that hyperpyrexia alone should not be accepted as a cure for early syphilis, but that it could be used as a powerful adjuvant to the usual chemotherapy with neoarsphenamine.

Many reports, including those made by Neymann and Phillips and Mundorff in America and by Batchelor, Thomson and Huggan in Great Britain have estab- 
lished the efficacy of combined pyretotherapy and chemotherapy in the treatment of neurosyphilis, interstitial keratitis and early optic atrophy, also of complicated and drug-resistant gonorrhoea. In the case of neurosyphilis, many years must elapse before the effect of this combined treatment can be decided. In complicated and persistent gonorrhoea the potency of the combined therapy has been adequately demonstrated to be greater than that of pyretotherapy alone.

In all the latest work, the necessary high and sustained temperature levels have been achieved by the use of air-conditioned fever cabinets in which the temperature is raised and maintained either by circulating hot moist air alone or by hot humid air plus induced electrical currents. As Neymann has emphasized, there is an essential and important difference between these two methods. In the former method, using hot moist air alone, the cabinet atmosphere must be at a higher temperature, say from $120^{\circ} \mathrm{F}$. to $140^{\circ} \mathrm{F}$., than that which is required when a shortwave high frequency oscillating current is used. Moreover, the rise of temperature attained by inductopyrexia, while sufficiently rapid, is less abrupt than in the unassisted conditioned air method, and the cabinet atmosphere is cooler throughout $\left(110^{\circ} \mathrm{F}\right.$.). Further, when the desired body temperature level, that is $106^{\circ} \mathrm{F}$. has been reached, the current may be switched off, and the patient induced to "coast" along on the pyrexial plateau in a less hot cabinet atmosphere. A falling temperature can be "boosted up " from time to time by switching on further increments of electrical energy. Electropyrexia enables the temperature inside the patient's body to be raised to high levels without the necessity of overheating the patient's skin ; it is a method of internal heating as opposed to the method of external heating by the non-electrical appliances. Neymann and others have shown that the inductotherm method is the more comfortable and is less exhausting to the patient because the pulse rate increases the more the skin is heated. It seems likely that some of the untoward effects such as anoxaemia and jaundice noticed in patients during and after treatment in non-electrical cabinets are attributable to the overheating of the skin inseparable from the use of high temperatures in the cabinet atmosphere.

What then is to be the place of pyretotherapy in the treatment of venereal diseases? In any balanced assessment it must be stressed that artificial fever is no panacea for uncomplicated gonorrhoea or early syphilis. This new procedure carries its own inherent limitations : it is costly, time-consuming for both patient and staff and requires expert nursing; therefore it is suitable for use only in large urban hospitals or in elaborate comprehensive private clinics.

As regards the type of apparatus, a consideration of the pros and cons seems to tilt the balance in favour of air-conditioned cabinets having an electrical inductotherm device for raising the body temperature by internal heating. With electrical internal heating, undesirable effects such as anoxaemia have not been observed, and in practice the administration of oxygen has not been required. Further, jaundice following inductopyrexia is rare unless chemotherapy with arsenicals is practised concurrently. With competent supervision and adequate preliminary examination of the patients to exclude those with some contra-indication such as tuberculosis, inductopyrexia would appear to be as safe as a general anaesthetic. Bowie, in Aberdeen, has not had any deaths in over four years' work. In the Edinburgh clinic in nearly four years of almost continuous operation there have not been any deaths among the many men treated, and but one death among the women. Whereas the risk to life in selected adult patients seems to be-low, this cannot be said of the extremes of age ; advanced age is a contra-indication and, in this sulphonamide era it is not justifiable to subject to electropyrexia babies with gonococcal ophthalmia or infants with vulvo-vaginitis. This lack of justification applies also to cases of uncomplicated gonorrhoea in adults. But in the indications quoted inductopyrexia may be the procedure of choice. For sulphonamideresistant gonorrhoea the combined use of sulphonamides and inductotherapy may have in the near future to yield pride of place to penicillin. 